\title{
Biologically-Inspired Massively-Parallel Architectures: A Reconfigurable Neural Modelling Platform
}

\author{
Steve Furber \\ University of Manchester
}

The SpiNNaker project aims to develop parallel computer systems with more than a million embedded processors. The goal of the project is to support large-scale simulations of systems of spiking neurons in biological real time. The architecture is generic and makes minimal assumptions about the network topologies that will be supported, the goal being to offer a fully reconfigurable platform to test hypotheses of brain function whether they arise from neuroscience, psychology, or elsewhere.

Steve Furber BE FRS FREng is the ICL Professor of Computer Engineering in the School of Computer Science at the University of Manchester. He received his BA degree in Mathematics in 1974 and his $\mathrm{PhD}$ in Aerodynamics in 1980 from the University of Cambridge, England. From 1980 to 1990 he worked in the hardware development group within the R\&D department at Acorn Computers Ltd, and was a principal designer of the BBC Microcomputer and the ARM 32-bit RISC microprocessor. He moved to the University of Manchester in 1990 where his research interests include low-power and asynchronous digital design and neural systems engineering. Steve was awarded a Royal Academy of Engineering Silver Medal in 2003, held a Royal Society Wolfson Research Merit Award from 2004 to 2009 and was awarded the IET Faraday Medal in 2007 and a CBE in 2008. He was a 2010 Millenium Technology Prize Laureate, and was awarded an Honorary DSc by the University of Edinburgh in 2010. 